\title{
Trituration and Fractal Dimension in Homeopathic Pharmacopoeia
}

Kalliantas D*, Kassalia ME and Karagianni CHS

Department of Materials Science and Engineering, School of Chemical Engineering, NTUA, Greece

*Corresponding author: Dimitris Kalliantas, Department of Materials Science and Engineering, School of Chemical Engineering, NTUA, 32 Keleou Street, Eleusis, Attica, Greece, Tel: 00306946170398; E-mail: dimitris@kalliadas.com

Received date: June 30, 2016; Accepted date: July 21, 2017; Published date: July 25, 2017

Copyright: ( $) 2017$ Kalliantas D, et al. This is an open-access article distributed under the terms of the Creative Commons Attribution License, which permits unrestricted use, distribution, and reproduction in any medium, provided the original author and source are credited.

\begin{abstract}
For many years now, homeopathy is considered to be an alternative and more physical way of treatment compared to traditional medicine. Though, little has yet been scientifically studied upon the matter. The scope of this paper is to investigate the relationship between the size-shape variations of eight starting Raw Solid Materials (RSM) and the role of trituration in the size-shape of these RSM's before they are turned into solutions. These materials are used in homeopathy as remedies, after successive grindings, before they are turned into homeopathic solutions. What is more, their fracture surface fractal dimension is investigated. The trituration process will be analyzed, which in homeopathic pharmacology leads to the substances having self-affined fractal structure regardless their type or hardness (metals, minerals, salts, shells, dry plants, etc.), a structure only depending on the size and shape of the powder grains of the starting RSM. The physical meaning of these results is that at the end of the process there is a gradual transformation of the size and shape of the original RSM during the trituration, depending on the physical condition of the materials before grinding them. In this paper, it is proved that, regardless of the starting RSM, the fixed trituration formalism used in Homeopathic Pharmacopoeia leads to a result which depends only on the powder grains size of the starting RSM.
\end{abstract}

Keywords: Trituration; Comminution; Fractals; Homeopathy Homeopathic remedies

\section{Introduction}

Homeopathy has recently drawn the attention as an alternative way of curing diseases, a way which appears to be more natural than traditional medicine. The drugs used in homoeopathic practice, and included in Pharmacopoeia, are originated usually from several substances and species of nature. The pharmaceutical processes of homoeopathy are characterized by a marvelous simplicity and perfection, requiring the greatest nicety and care, so that the homoeopathic designation is characterized by special degree fineness in quality.

Looking over homeopathic medicinal texts, the words "potency", "potentization", "dilution", and "solution" are synonymous with the terms "attenuation" or "trituration". By decision of the Pharmacopoeia Convention, the official terms are "attenuation" for liquids and "trituration" for solids materials [1]. Trituration is the preparation of a drug, introduced by S. Hahnemann, by which the finely powdered medical substance is grounded for a certain time in a mortar with a pestle combined with a certain proportion of sugar of milk (lactose) [2]. There are two main traditional preparation scales for all medical homeopathic substances. The first is the decimal scale (containing $1 / 10$ of the original substance) and the second is the centesimal one (containing 1/100 of the original substance). In the process, respecting the scale of trituration, a progressive division and diminution of the solid medical substance takes place through mechanical subdivision. So, the visible particles of the substance become gradually transformed as the number of triturations ascends. During trituration, the fragmentation of RSM may be random, but depends on several different factors that will not be discussed in this study.
The term fractal was conceived by Mandelbrot and it derives from the Latin fractum, meaning something that is broken down into uneven pieces sharing a common trait, the fractal dimension. Although the word "fractal" is of recent origin, the notion of fractal geometry is not new. In fact, there is a geometric measure theory of sets of integral and fractional dimension, sometimes referred to as Hausdorff or Hausdorff-Besicovitch dimension. It should be mentioned that, much of the mathematical work in this area often contains high ingenious arguments, due to Besicovitch [3].

Dimension is an exponent characterizing how some aspect-mass, e.g. the number of boxes in a covering, of an object change with the size of the object. Although a universally accepted definition is yet to be found, fractals constitute a primary, consequently non-definable concept in geometry, much like the force in Physics [4-6]. The mathematical foundations of fractal geometry slip outside the scope of this paper, so for further examination on the matter the reader is kindly advised to turn to the references [7-9]. Only one conceptually simple technique is essentially needed to be described in order to discuss the fractal features of the RSM's fractured surfaces after trituration. The applied technique is suggested by Mandelbrot et al. in a paper, where he introduced the main ideas and methods of the fractal analysis to the metallographic community [10]. Different experimental techniques have been used over the years in the fractal analysis parallel to numerical simulations of the fracture process. That process has gained popularity and it has become a very useful tool of analysis. Details on this subject can be found from the reader in the references [11-19]. One of the difficulties in understanding the different variations when measuring the fractal dimensions are the methods of measuring used in different studies. In other words, part of the problem derives from the existence of a large variety of experimental techniques that are combined with insufficient analysis of their comparative merits [20]. Another problem that relates to the universal nature of the results in trituration is the possible dependence of the 
fracture-roughness exponent $\zeta$ on the specific material under study. A self-affined surface $z(x, y)$, isotropic in the $(x, y)$-plane, is invariant under the scale transformation $\left(\lambda \mathrm{x}, \lambda \mathrm{y}, \lambda^{3} \mathrm{z}\right)$ where $\zeta$ is defined as the roughness exponent. It has been proved in many occasions that fractured surfaces statistically exhibit self-affined scaling properties. There are results which indicate that the scaling properties of the fractured surface are not universal, but depend, albeit weakly, on the material's properties. While values of the roughness exponent $\zeta$ are independent from dimension, differences in the fractured morphology and in the breakdown characteristics are observed [21]. The difficulty in applying the conventional approach is that in all methods only twopoint correlations are analyzed to find the fractal dimension or the roughness exponent. This parameter is currently used as the only quantitative characteristic of the surface. Correspondingly, there appears to be no mathematical method to distinguish two surfaces with similar fractal dimensions and different morphologies. Note that such distinction is easily achieved by human eye and brain, which hints towards the missing link in the extensive attempts to find a relationship between the fractal morphology of the surface and the mechanical properties of the material.

Firstly, there has to be a separation between a self-similar and a selfaffined fractal. Self-affined fractal is a shape consisting of smaller copies of it; all scaled by affinities and linear transformations with different contraction ratios in different directions. Self-similar fractal is a shape consisting of smaller copies of it; all scaled by similitude, linear transformations with the same contraction ratios in every direction. Nevertheless, we are able to define as fractal any subtotal $\mathrm{w}$, in geometry is a subtotal of $\mathrm{Rn}$, which displays self-similarity or selfaffinity to the primal one (mother) and whose fractal dimension does not exceed the topological one. Although it is true that on many occasions mathematical fractals prove impressive, the same does not apply in nature's creations. These creations are formed through chaotic processes, during which an endless number of "pieces" get interconnected in a quite perplexed way, disproving a mathematical simulation created by a repetitious algorithm. Therefore, natural fractals possess a personality of certain depth and mysterious qualities that no algorithm, even non-linear, is able to faithfully reproduce.

The basis of fractal analysis are events occurring in the same types of distribution on different scales of observation, and most fractal analysis methods are using means of scaling in order to determine the fractal relation [6].

By expressing the degree of complexity, the Fractal Dimension (FD) is a useful tool to describe natural objects and, even, procedures [6]. One popular approach for obtaining the FDs, as a measurement of the space filling property of an object, is the Box-Counting Method (BCM). This method will be used in the samples studied, with the help of NARO. The main process of the BCM is to fully cover a planar object with $\mathrm{N}$ boxes, with each side being of length $\lambda$, which is also the scale of measurement. We assume, thus, having a small quantity of RSM before trituration, referred to as X, immerged into a fully 2dimension surface in flattening to form a flat surface, as illustrated in Figure $1 . \mathrm{L}$ is the length of the surface and $\lambda$ the length of the smaller $\lambda<\mathrm{L}$ boxes that form the grid. We set as $\mathrm{N}(\mathrm{L}, \lambda)$ the minimum space of "boxes" on the $\lambda$ side that fully cover our planar mass. In our example, we assume that $N(L, \lambda)=10$ or $N(L, \lambda)=100$.

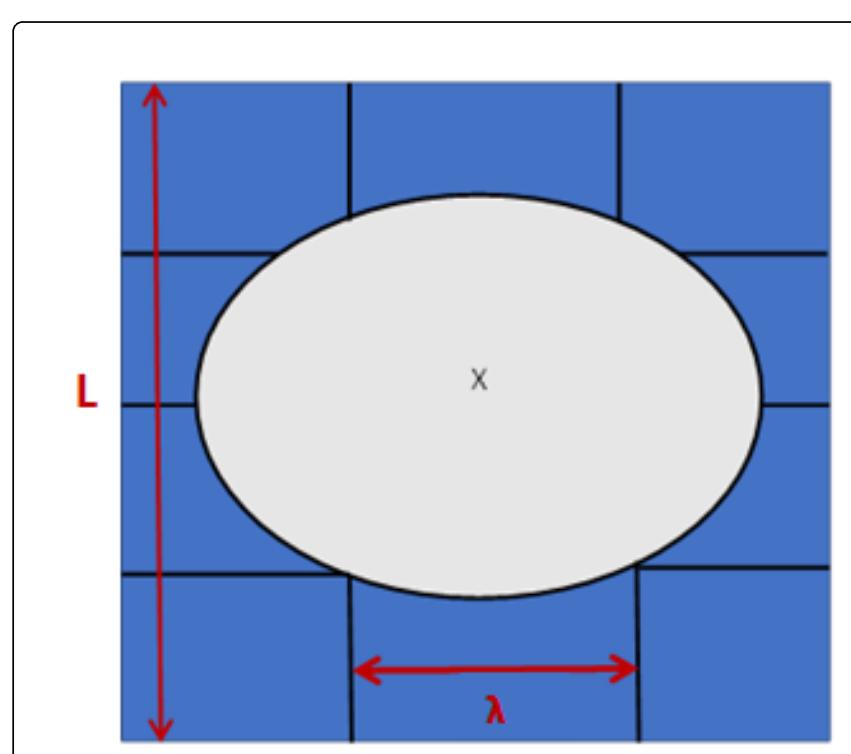

Figure 1: Separation of a small quantity (droplet) of an RSM in ten "boxes" with $\lambda$ length in a surface of length $L$.

If $\lambda$ is large, the above covering cannot fill the "blanks" correctly inside the mass or the indentions on the boundaries of the smaller $\lambda$. Thus, in order to geometrically describe the mass's structure, it must be $\lambda \rightarrow 0$ and $\mathrm{N}$ (L, $\lambda) \rightarrow \infty$, for its " $\delta$-measure" to be $M_{\delta}=N(L, \lambda) \cdot \lambda^{\delta}, \delta>0$ and finite.

\section{The index $\delta$ analysis:}

If the mass is compact, after being enlarged, it somehow stops presenting structure details so it becomes clear that $\mathrm{N} \sim(\mathrm{L}, \lambda) 2$. Consequently, the dimension of the space in which it was immerged results in $\delta=\mathrm{d}=2$. If we assume not knowing the $\delta$ value, ensuring $\mathrm{M} \delta<\infty$, and we attempt to estimate it with the following procedure, then:

$$
M_{\delta}\left\{\begin{array}{l}
0 \\
0 \\
\infty
\end{array} \leq c \leq \infty\right.
$$

So we define $\quad D_{0}=\underset{\delta}{\operatorname{Inf}}\left\{\delta / M_{\delta}=0\right\}=\operatorname{Sup}\left\{\delta / M_{\delta}=\infty\right\}$

(Inf, Sup are measuring topologies). It is also defined as morphofractal or as the fractal dimension of the mass. If $\mathrm{d}=2$ equals the immersion dimension, which means the minimum integer dimension of the Euclidean space fully including the mass, we are going to prove that

Since $N(L, \lambda) \leq\left(\frac{L}{\lambda}\right)^{\delta}$ and $N(L, \lambda)=c \lambda^{-D_{0}}, \lambda \rightarrow 0$, we deduce that $c \leq L^{d} \lambda^{D_{0}-d} \Rightarrow D_{0} \leq d$, so the c constant will not tend towards zero for $\lambda \rightarrow 0$. "De-dimentionalising" the "length" units that were used we set $\varepsilon=\frac{\lambda}{L}$, then with $\mathrm{N}(\mathrm{L}, \lambda)=\mathrm{N}(\varepsilon)$ we have or

$$
N_{(\varepsilon)}=c \cdot \varepsilon^{-D_{0}}, \varepsilon \rightarrow 0
$$


Through which the fractal dimension $\mathrm{D}_{0}$ is defined, where $\mathrm{c}>0$, is an unknown constant depending on the related problem [22]. All fractured sets presenting a related self-similarity or self -affinity have a fractal dimension. A X set as e.g. the mother RSM until turning it into a homeopathic solution, during trituration, presents a related selfaffinity, if it can be subdivided into $\mathrm{K}$ equivalent subsets, every one of which enlarged at an $\mathrm{M}$ fixed factor gives $\mathrm{X}$. The homeopathic remedies -from mother RSM to its final form- are relatively self-affined during the trituration and dilution phase because they are homogeneous (single-phase) and can be subdivided into equivalent parts, every one of which enlarged at a fixed factor, gives the initial one. If we took $10 \mathrm{~g}$ of mother RSM to prepare a homeopathic remedy, $1 \mathrm{~g}$ would be triturated into $9 \mathrm{~g}$ or $99 \mathrm{~g}$, depending on the scale, of pure sugar of milk. From the new mass, we take $1 \mathrm{~g}$ and triturate it into $9 \mathrm{~g}$ or $99 \mathrm{~g}$ of pure lactose and we follow the same process/procedure for $\mathrm{k}$ triturations (Table 1).

\begin{tabular}{|l|l|l|}
\hline Triturations & Factor & Remaining Quantities \\
\hline $\mathrm{n}=1$ & $\varepsilon 1=1 / 10$ & $\mathrm{~N} 1=9$ \\
\hline $\mathrm{n}=2$ & $1 / 102$ & $\mathrm{~N} 2=92$ \\
\hline $\mathrm{n}=3$ & $\varepsilon 3=1 / 103$ & $\mathrm{~N} 3=93$ \\
\hline $\mathrm{n}=\mathrm{K}$ & $\varepsilon \mathrm{k}=1 / 10 \mathrm{k}$ & $\mathrm{NK}=9 \mathrm{~K}$ \\
\hline
\end{tabular}

Table 1: The process of the decimal trituration.

In case of a centesimal trituration, then all the aspects apply correspondingly, as showed in Table 2.

\begin{tabular}{|l|l|l|}
\hline Triturations & Factor & Remaining Quantities \\
\hline $\mathrm{n}=1$ & $\varepsilon 1=1 / 100$ & $\mathrm{~N} 1=99$ \\
\hline $\mathrm{n}=2$ & $1 / 1002$ & $\mathrm{~N} 2=992$ \\
\hline $\mathrm{n}=3$ & $\varepsilon 3=1 / 1003$ & $\mathrm{~N} 3=993$ \\
\hline $\mathrm{n}=\mathrm{k}$ & $\varepsilon \mathrm{k}=1 / 100 k$ & $\mathrm{Nk}=99 k$ \\
\hline
\end{tabular}

Table 2: The process of the centesimal trituration.

And generally, if we use triturations with:

$\varepsilon 1=\frac{1}{10}, \varepsilon 1=\frac{1}{10^{2}} \ldots \ldots \ldots \ldots \ldots . . . \varepsilon 1=\frac{1}{10^{\kappa}}$

Then,

$$
\begin{aligned}
& \lim _{k \rightarrow \infty} \frac{\log \left(10^{k}-1\right)^{n}}{\log \left(10^{k}\right)^{n}}=\lim _{k \rightarrow \infty} \frac{n \log \left(10^{k}-1\right)}{n \log 10^{k}}=\lim _{k \rightarrow \infty} \frac{\log \left(10^{k}-1\right)}{k \log 10} \\
& =\lim _{k \rightarrow \infty} \frac{\log \left(10^{k}-1\right)}{k}=1
\end{aligned}
$$

According to the definition, the fractal dimension of an $\mathrm{X}$ set is defined as the number [23-25]:

$$
D=\frac{\log \kappa}{\log M}
$$

Where, $\kappa:$ Is the number of the equivalent parts in which $\mathrm{X}$ is subdivided into, and $\mathrm{M}$ : Is the enlargement coefficient of each one. Or generally:

$$
D=\frac{\log (N)}{\log \left(\frac{1}{r}\right)}
$$

Where $\mathrm{N}$ is the number of fragments, and $\mathrm{r}$ represents the ratio of similarity. Equation (6) may be rewritten as followed:

$$
D=\frac{-\sum_{1} \frac{1}{N}}{\log \left(\frac{1}{r}\right)} \log \left(\frac{1}{N}\right)
$$

D en o t in $P_{i}=\frac{1}{N}$, which is the occupation probability of every

$$
\text { fragment, eq. (7) is rewritten as: } D=\frac{S}{\log \left(\frac{1}{r}\right)} \text { (8) }
$$

Where $S=-\Sigma P^{i} \log P^{i}$ is Shannon (Information) Entropy?

While it is a tradition in materials science to search for global relations, the quest in this context is usually correlations between measured quantities and mechanical properties and not between measured quantities and formalism of processes which lead to fractured surfaces. Although many experiments are carried out along these lines, the results are inconclusive [20]. Mandelbrot et al. first showed that a fractured surface is a fractal in nature and suggested that the fractal dimension of the surface is a measure of toughness in metals in 1984 [10]. However, experimental investigations [3,10,14,25-27] showed negative correlation between fractal dimension and toughness of materials, which is in contrary to the above-mentioned belief. Nevertheless, there is a general agreement in the community of material scientists stating that the higher the fracture toughness, the rougher the fracture surface, which may correspond to higher fractal dimension. However, at present this conjecture is yet to be substantiated. A universal fractal behavior poses a challenge to explain such a fundamental law of nature and a universal fractal dimension should be independent of material characteristics. But, there are stable products' manufacturing methodologies, as is trituration for solids and attenuation for liquids, such as in production of homeopathic remedies (all cases are the same formalism), where the final product is a part of the division of an RSM with fixed formalism manufacturing through a broken surfaces process. Here, a universal fractal dimension, independent of the type of materials, would be a useful tool as a proof of the existence of original RSM material at all stages of manufacture up to the highest dilutions and this follow is similar to the above approach.

Thus, the universal fractal dimension of the process for the decimal trituration may be:

$$
D_{o \mu}=\frac{\log 9^{n}}{\log 10^{n}}=\frac{\not \not \log 9}{\not 2 \log 10}=0,9542425
$$

Whereas for the centesimal trituration may be:

$$
D_{o \mu}=\frac{\log 99^{n}}{\log 100^{n}}=\frac{\not \not \log 99}{\not\lfloor\log 100}=0,99781759
$$

These numbers are irrational which means that entropy is indicated. Entropy quantifies the diversity, uncertainty, or randomness of a system. Entropy is the measure of disorder in physical systems. Also, they mean that the decimal or centesimal set of starting RSM, which is immerged into a $\mathrm{d}=2$-dimension space, does not consist of individual parts (molecules, atoms, aggregates, coordination compounds etc.) before turning it in homeopathic solutions, otherwise its dimension would have been $\mathrm{Do}=0$. On the other hand, though, it does not consist of a whole mass, since Do $\mu<1$ and the total Lebesque measure of the 
set is 0 , resulting from the existence-uniqueness theorems on the filling of metric spaces eq. $[11,12]$.

$$
\ell=\lim _{n \rightarrow \infty} N_{n} \varepsilon_{n}=\lim _{n \rightarrow \infty}\left(\frac{9}{10}\right)^{n}=0
$$

or

$$
\ell=\lim _{n \rightarrow \infty} N_{n} \varepsilon_{n}=\lim _{n \rightarrow \infty}\left(\frac{99}{100}\right)^{n}=0 .
$$

This means that by grinding, qualitative transformation of original RSM possibly begins.

\section{Methods and Materials}

We studied mathematically and experimentally a part of Hahnemann's trituration method that has to do with the correlation between the grain size of the RSM and the final form of the RSM after the trituration and before turning it into a solution. At least one hour should be consumed to produce each trituration. One hour for this process, however, was S. Hahnemann's rule. This principle was presented by him. In our samples, we adopted the decimal scale, and thus a better preparation was ensured, since every decimal trituration requires double the time for grinding, and this method is therefore universally adopted. Each potency is triturated for at least one hour, so the sixth trituration demands no less than six hours of constant triturating. There is no need or any advantage in doing so beyond the third centesimal or sixth decimal trituration, since Hahnemann proved conclusively-and clinical experience fully verified that fact-that beyond these potencies all homeopathic medicines can be easily prepared in a liquid state. Our samples were prepared by hand, according to the decimal scale. The apparatus required is:

An unglazed by rubbing with wet sand, sterilized mortar and pestle.

A sterilized scraper (porcelain spoon), a measuring tile, a timer and a balance (kern S72, version 4.07-centimeter accuracy $0.00 \mathrm{~g}$ ).

Camera Canon EOS 600D with lens EX SIGMA 105 mm1:2.8 DG MACRO and ring light flash NISSIN DIGITAL MF18 MACRO used for taking photos of the materials after their flattening to form a flat surface of unit thickness.

\section{SEM (SEM Quanta 200, FEI).}

To determine the fractional dimension of our samples we used software for fractal analysis for Windows from the National Agriculture and Food Research Organization (hereafter referred to as NARO) 3-1-1 Kannondai, Tsukuba, Ibaraki 305-8517, Japan.

Photoshop Adobe Creative Suite version CS6 for Windows.

Firstly, it is useful to have a compilation of the available experimental data. Usually, and especially in this software, images from Camera, SEM, TEM, etc. photos they are used. These data sets are used, firstly to investigate the relationship of the original grain size with the final fractal form, secondly to examine the fractal relation in two or three-dimensional space, and finally to attempt to understand the relationship between the patterns of the different rupture modes caused by a stress field. The SEM pictures were processed with Photoshop Adobe Creative Suite version CS6, in order to remove lactose from the background of the RSMs.

One part of the drug substance and 9 parts of the sugar of milk is taken. The sugar of milk is divided into three parts in the ratio of 1:3:5 on a measuring tile. The process of trituration takes one hour. The entire process is divided into three stages. Each stage lasts twenty minutes. In each stage, one part of sugar of milk is added. Each stage is divided into two sub-stages those last ten minutes each. The process carried out in the first ten minutes of each stage is repeated for the next ten minutes. Each sub-stage of ten minutes consists of grinding or pulverizing for six minutes and scraping and mixing for four minutes. Trituration is done for six minutes with pestle fully pressed down with a firm grip and thumb on top. It is firmly moved in anticlockwise direction, in a circular direction, going from center to periphery and back from periphery to center without lifting the hand but by controlling the movement from the wrist joint. The mixture is then thoroughly scraped from the walls of the mortar with a spatula and mixed together uniformly.

Sugar of milk is separated in this way, so that there is a homogenous and uniform mixing of the drug with the lactose. The trituration is then carried out with the three parts of sugar of milk, as directed. This is preserved with a label mentioning the name of the remedy with the potency. In all potencies, up to $6 \mathrm{X}$, one part from the weight of the preceding potency is triturated for one hour with nine parts from the weight of sugar of milk, as directed, to give the desired potency. For preparing centesimal attenuations, one (1) part of the medicinal raw material is triturated with ninety-nine (99) parts of sugar of milk (lactose monohydrate). Then, we triturate for a sufficient time. The result is the 1C trituration. One (1) part of the 1C trituration is triturated with ninety-nine (99) parts of lactose monohydrate. Trituration for a sufficient time follows. The result is the $2 \mathrm{C}$ trituration. Finally, one (1) part of the $2 \mathrm{C}$ trituration is triturated with ninety-nine (99) parts of lactose monohydrate. After triturating for a sufficient time, the $3 \mathrm{C}$ trituration is formed. All these are afterwards used to form our final homeopathic solutions. In our experimental process, different commercial materials in different forms are used for this purpose, which are obtained from various Raw Starting Materials.

\section{Materials}

Arnica in a dried plant form was given by M. Filianu's pharmacy supply. Because the material was initially in a fibrous form, the sample was triturated for six minutes and scraped for four minutes without lactose in a mortar.

Hypericum in a dried stem and leaf form was given by $\mathrm{A}$. Chatzopulu's pharmacy supply. Because of its fibrous form, the above procedure as in Arnica was followed.

Sodium chloride powder for household purposes in a form of granules. Benzoic acid in granules was given by Alfa Aesar GmbH Germany (purity 99\%). Sulfur, in a form of fine powder sulfur flowers, was given by Sigma-Aldrich GmbH Germany. Calcium phosphate in extra fine powder was given by Sigma-Aldrich $\mathrm{GmbH}$ Germany (purity 96\%). Calcium carbonate by scraping the inner of an oyster's surface. Because the sample was not uniform, it was grinded for six minutes and scraped-।for four minutes without lactose in a mortar. Iron in extra fine shavings was given by Ferac GmbH Germany (highest chemical purity $>98 \%$ ).

Sugar of Milk commercial powder was given by B. Kaliva's pharmacy supply. Sugar of milk is a product of animal life, one of the constituents of milk, obtained by evaporating the whey of the milk. It is of pure white color, faintly sweet taste and odourless. For homoeopathic use, it ought to be re-crystallized, as the ordinary commercial product is not always perfectly pure. It must be kept in a 
Citation: Kalliantas D, Kassalia ME, Karagianni CHS (2017) Trituration and Fractal Dimension in Homeopathic Pharmacopoeia. Pharm Anal Acta 8: 554. doi:10.4172/2153-2435.1000554

Page 5 of 12

dry place, as it becomes musty when exposed to dampness. No other known substance could reform hard mineral substances from sharp, flinty crystals to an inconceivably fine powder, so that they can be absorbable by the body. Also, its preservative properties are great, keeping the minute particles of triturated metals untarnished by oxidation. In our samples, for preparation $1 \mathrm{X}$ potency used 1 part of 2 $\mathrm{g}$ of RSM and 9 parts of sugar of milk distributed as shown in Table 3. To prepare the $2 \mathrm{X}$ potency until the last $6 \mathrm{X}$, we used 1 part of $2 \mathrm{~g}$ of ground obtained from every previous potency and 9 parts of sugar of milk distributed as shown in Table 4.

\begin{tabular}{|l|l|l|l|l|l|l|l|l|}
\hline Quant. Gr & Arnic & Hypericum & Sod. chlor. & Benz. acid & Sulfur & Calc. phos. & Calc. car. & Iron \\
\hline RSM & 2 & 2 & 2 & 2 & 2 & 2 & 2 & 2 \\
\hline Lactose & $1.2=2$ & $1.2=2$ & $1.2=2$ & $1.2=2$ & $1.2=2$ & $1.2=2$ & $1.2=2$ & $1.2=2$ \\
\hline Lactose & $3.2=6$ & $3.2=6$ & $3.2=6$ & $3.2=6$ & $3.2=6$ & $3.2=6$ & $3.2=6$ & $3.2=6$ \\
\hline Lactose & $5.2=10$ & $5.2=10$ & $5.2=10$ & $5.2=10$ & $5.2=10$ & $5.2=10$ & $5.2=10$ & $5.2=10$ \\
\hline
\end{tabular}

Table 3: Quantities for 1X trituration.

\begin{tabular}{|l|l|l|l|l|l|l|l|l|}
\hline Quant. Gr & Arnica & Hyperic & Sod. chl & Benz. acid & Sulfur & Calc. PH. & Calc. CA. & Iron \\
\hline RSM & 2 & 2 & 2 & 2 & 2 & 2 & 2 & 2 \\
\hline Lactose & $1.2=2$ & $1.2=2$ & $1.2=2$ & $1.2=2$ & $1.2=2$ & $1.2=2$ & $1.2=2$ & $1.2=2$ \\
\hline Lactose & $3.2=6$ & $3.2=6$ & $3.2=6$ & $3.2=6$ & $3.2=6$ & $3.2=6$ & $3.2=6$ & $3.2=6$ \\
\hline Lactose & $5.2=10$ & $5.2=10$ & $5.2=10$ & $5.2=10$ & $5.2=10$ & $5.2=10$ & $5.2=10$ & $5.2=10$ \\
\hline
\end{tabular}

Table 4: Quantities for 2X until the last 6X triturations.

\begin{tabular}{|c|c|c|c|}
\hline Name & RSM & $3 x$ & $6 x$ \\
\hline Arnica & & & \\
\hline W, H & 544,362 & 496,457 & 496,457 \\
\hline C OR BD & 100,0 & 39,1 & 26,3 \\
\hline$R(n)$ & $-0,9999(8)$ & $-1,0000(5)$ & $-0,9998(4)$ \\
\hline FD & 1,9606 & 1,7021 & 1,4785 \\
\hline BS & Co & Co & Co \\
\hline 1 & 196928 & 88715 & 59649 \\
\hline 2 & 49232 & 27760 & 22313 \\
\hline 4 & 12376 & 8648 & 8002 \\
\hline 8 & 3128 & 2652 & 2757 \\
\hline 16 & 782 & 787 & \\
\hline 32 & 204 & & \\
\hline 64 & 54 & & \\
\hline 128 & 15 & & \\
\hline 256 & & & \\
\hline 512 & & & \\
\hline
\end{tabular}

Table 5: Arnica results. 
Citation: Kalliantas D, Kassalia ME, Karagianni CHS (2017) Trituration and Fractal Dimension in Homeopathic Pharmacopoeia. Pharm Anal Acta 8: 554. doi:10.4172/2153-2435.1000554

\begin{tabular}{|c|c|c|c|}
\hline Name & RSM & $3 x$ & $6 X$ \\
\hline Hypericum & & & \\
\hline W, H & 544,362 & 496,457 & 496,457 \\
\hline C OR BD & 100,0 & 42,0 & 24,7 \\
\hline$R(n)$ & $-0,9999(8)$ & $-0,9998(5)$ & $-0,9986(4)$ \\
\hline FD & 1,9606 & 1,6957 & 1,4124 \\
\hline BS & Co & Co & Co \\
\hline 1 & 196928 & 95109 & 55941 \\
\hline 2 & 49232 & 31270 & 23435 \\
\hline 4 & 12376 & 9706 & 8885 \\
\hline 8 & 3128 & 2972 & 2957 \\
\hline 16 & 782 & 865 & \\
\hline 32 & 204 & & \\
\hline 64 & 54 & & \\
\hline 128 & 15 & & \\
\hline 256 & & & \\
\hline 512 & & & \\
\hline
\end{tabular}

Table 6: Hypericum results.

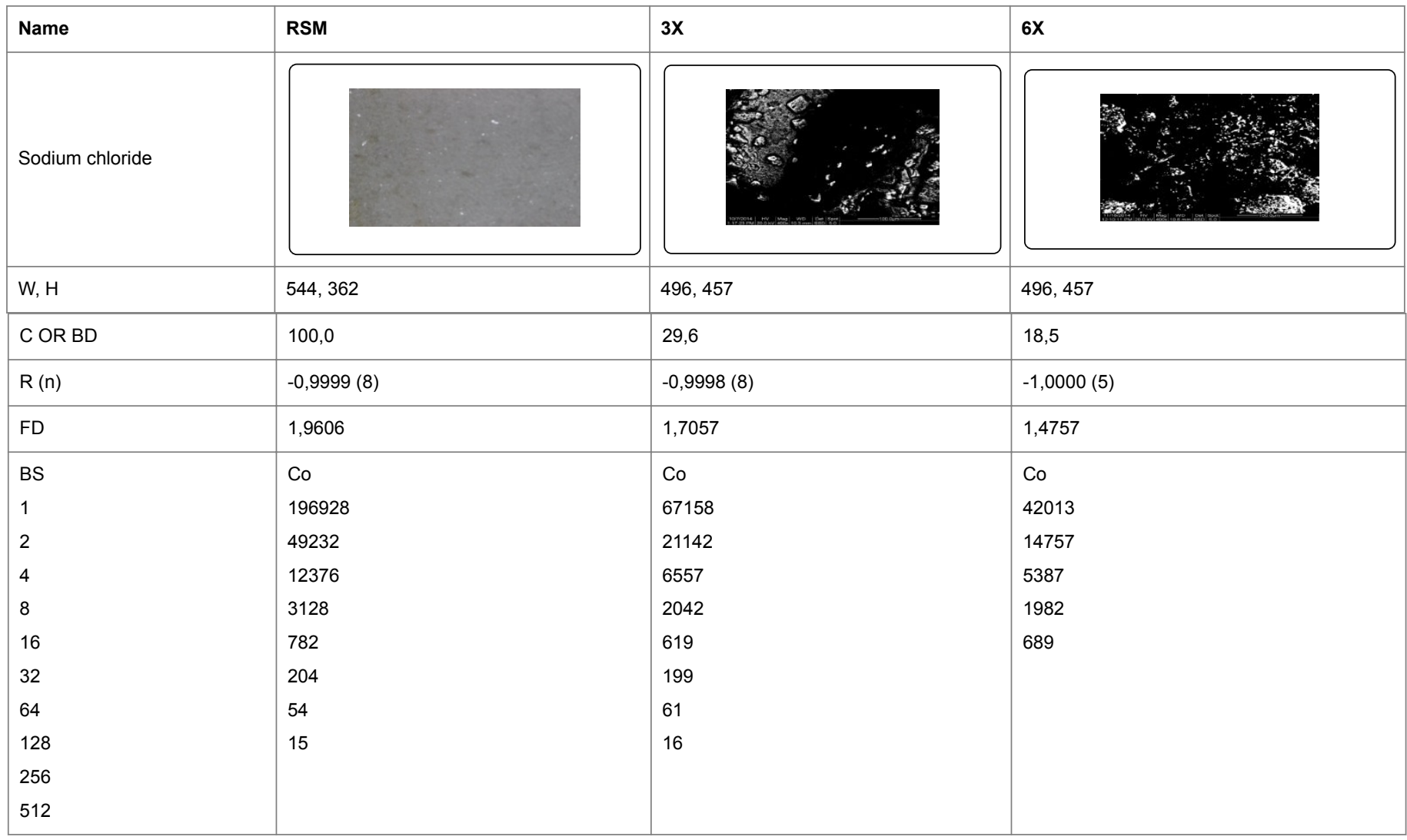

Table 7: Sodium chloride results. 
Citation: Kalliantas D, Kassalia ME, Karagianni CHS (2017) Trituration and Fractal Dimension in Homeopathic Pharmacopoeia. Pharm Anal Acta

\section{Discussion}

After trituration, a random bit of amount of each triturated sample by $3 \mathrm{X}$ and $6 \mathrm{X}$ were plated and photographed under a SEM. Tables 5-12 show: RSM photo images.

SEM images of $3 \mathrm{X}$ and $6 \mathrm{X}$ trituration of our substances and their distributions after processing with Photoshop. Their analysis to determine the fractal dimension after processing with NARO software

\section{The Analysis Results}

G: Glossary: W: Width; H: Height; C: Cover; B: BrightDiff; R: Correlation coefficient; (n): Number of data to calculate; FD: Fractal Dimension; BS: Box size; Co: Count.

\begin{tabular}{|c|c|c|c|}
\hline Name & RSM & $3 x$ & $6 X$ \\
\hline Sulfur & & & \\
\hline W, H & 544,362 & 497,457 & 496,457 \\
\hline C OR BD & 100,0 & 26,5 & 22,2 \\
\hline$R(n)$ & $-0,9999(8)$ & $-0,9997(4)$ & $-0,9994(4)$ \\
\hline FD & 1,9606 & 1,4315 & 1,3825 \\
\hline BS & Co & Co & Co \\
\hline 1 & 196928 & 60309 & 50244 \\
\hline 2 & 49232 & 23080 & 20358 \\
\hline 4 & 12376 & 8771 & 8021 \\
\hline 8 & 3128 & 3048 & 2810 \\
\hline 16 & 782 & & \\
\hline 32 & 204 & & \\
\hline 64 & 54 & & \\
\hline 128 & 15 & & \\
\hline 256 & & & \\
\hline 512 & & & \\
\hline
\end{tabular}

Table 8: Flowers of sulfur results.

In Figure 2 we see a progressive reduction of the FD during triturations and proportionality with respect to the initial form that does not depend on the roughness of substances but on the previous physical conditions (granules, powder, fine powder, etc.). From the above we deduce that the mother RSM set after trituration, before turning it into homeopathic solutions, behaves like a Cantor C1/10 or $\mathrm{C} 1 / 100$ set. It is a closed and bounded set that can be considered perfect, as each part of it constitutes the marginal point of the set formed by a dense and not absolutely countable set of elements (molecules, ions, agglomerates, coordination compounds etc.). Cantor sets can be understood geometrically by imagining the continuous removal of a set portion of a shape in such a way that at every stage of removal, the chucks of the shape that remain each have the same percentage removed from their centers. If this removal process continues infinitely, then the tiny bits of the shape that remains form a Cantor set. It is a set formed in an inductive way, and according to Kolmogorov, it could also define the probability of a contingencyproportionally to the measure concept of a set-like a real set function-a function having as independent variable a set-fulfilling certain conditions [28-30]. Our materials were a small sample out of the natural ones used by homeopathic pharmacopoeia. They were two dry plants (Arnica, Hypericum), two mineral substances (calcium phosphate, sulfur); one oyster (calcium carbonate), one metal (iron), one solid organic acid (benzoic acid) and one salt (sodium chloride). 
Citation: Kalliantas D, Kassalia ME, Karagianni CHS (2017) Trituration and Fractal Dimension in Homeopathic Pharmacopoeia. Pharm Anal Acta 8: 554. doi:10.4172/2153-2435.1000554

Page 8 of 12

\begin{tabular}{|c|c|c|c|}
\hline Name & RSM & $3 x$ & $6 x$ \\
\hline Benzoic acid & & & \\
\hline $\mathrm{W}, \mathrm{H}$ & 544,362 & 496,457 & 496,457 \\
\hline C OR BD & 100,0 & 52,1 & 16,1 \\
\hline$R(n)$ & $-0,9999(8)$ & $-0,9997(4)$ & $-1,0000(6)$ \\
\hline FD & 1,9606 & 1,6964 & 1,4694 \\
\hline BS & Co & Co & Co \\
\hline 1 & 196928 & 118007 & 36509 \\
\hline 2 & 49232 & 38486 & 13031 \\
\hline 4 & 12376 & 12015 & 4742 \\
\hline 8 & 3128 & 3453 & 1743 \\
\hline 16 & 782 & & 630 \\
\hline 32 & 204 & & 220 \\
\hline 64 & 54 & & \\
\hline 128 & 15 & & \\
\hline 256 & & & \\
\hline 512 & & & \\
\hline
\end{tabular}

Table 9: Benzoic acid results.

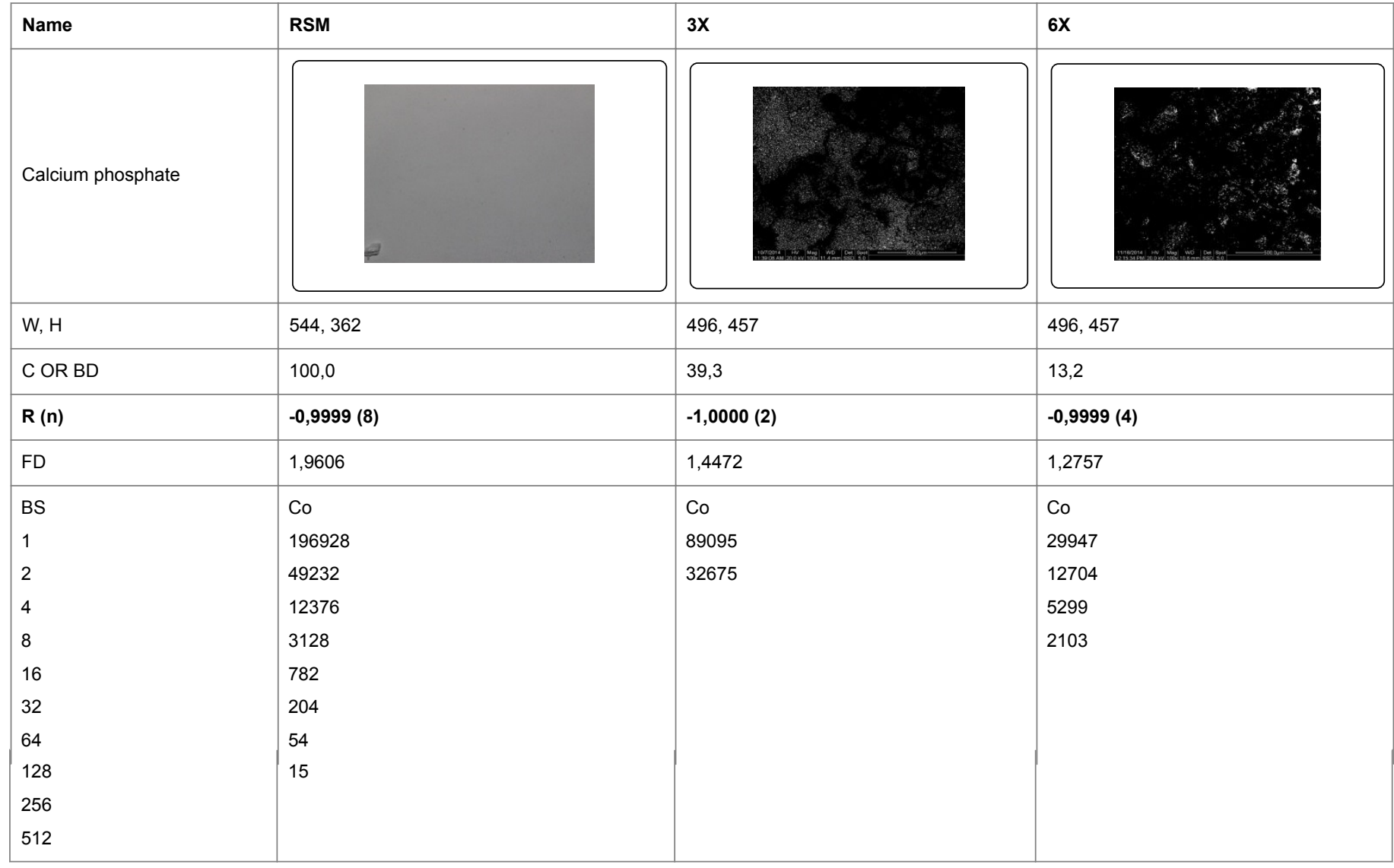

Table 10: Calcium phosphate results. 
Citation: Kalliantas D, Kassalia ME, Karagianni CHS (2017) Trituration and Fractal Dimension in Homeopathic Pharmacopoeia. Pharm Anal Acta 8: 554. doi:10.4172/2153-2435.1000554

Page 9 of 12

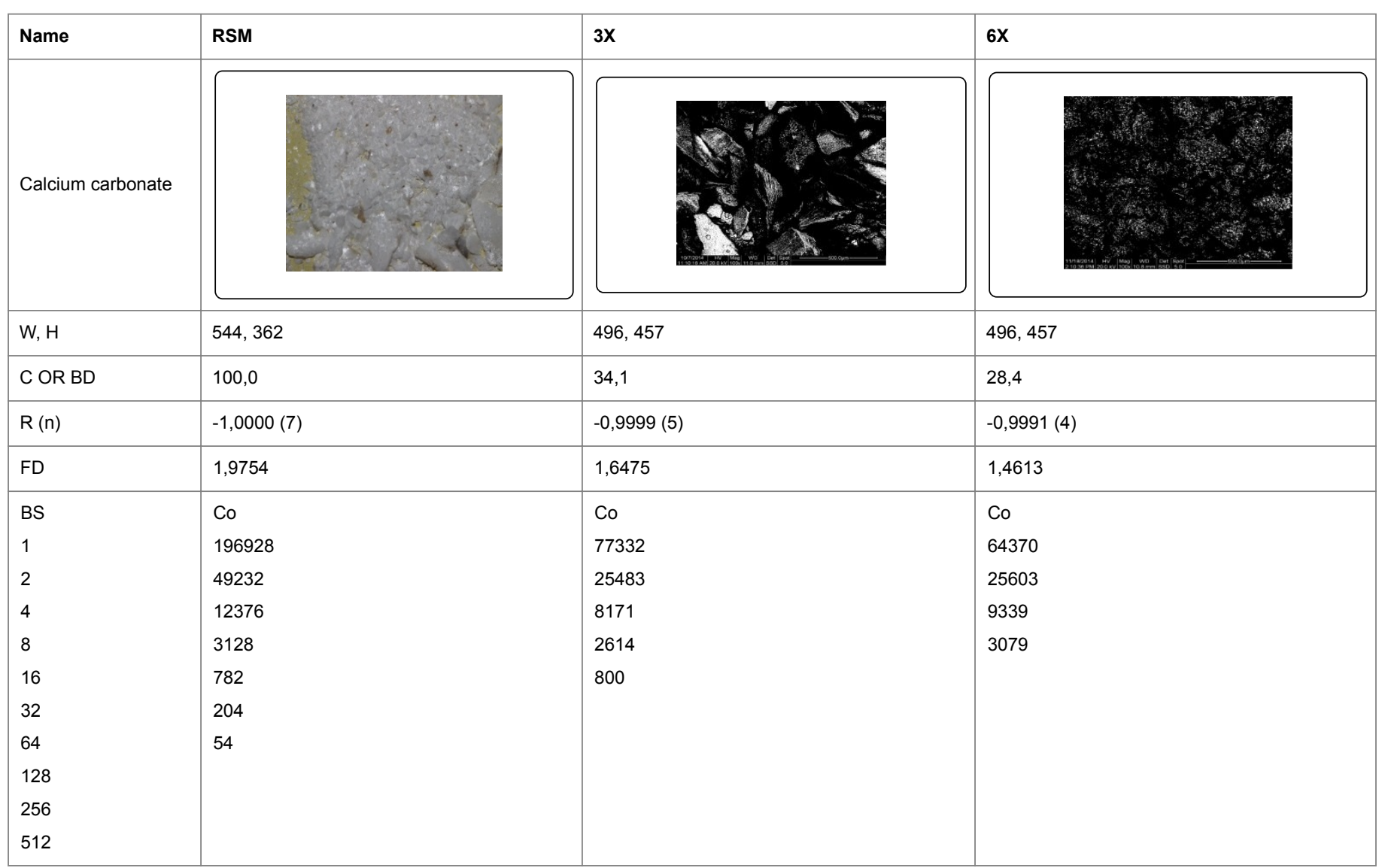

Table 11: Calcium carbonate results.

\begin{tabular}{|c|c|c|c|}
\hline Name & RSM & $3 x$ & $6 X$ \\
\hline Iron & & & \\
\hline $\mathrm{W}, \mathrm{H}$ & 544,362 & 496,457 & 922,720 \\
\hline C OR BD & 100,0 & 18,1 & 28,9 \\
\hline$R(n)$ & $-0,9999(8)$ & $-1,0000(2)$ & $-0,9992(3)$ \\
\hline FD & 1,9606 & 1,2618 & 1,2464 \\
\hline BS & Co & Co & Co \\
\hline $\begin{array}{l}1 \\
2 \\
4 \\
8 \\
16 \\
32 \\
64 \\
128 \\
256 \\
512\end{array}$ & $\begin{array}{l}196928 \\
49232 \\
12376 \\
3128 \\
782 \\
204 \\
54 \\
15\end{array}$ & $\begin{array}{l}40963 \\
17083\end{array}$ & 1918518580234085 \\
\hline
\end{tabular}

Table 12: Iron results. 
Citation: Kalliantas D, Kassalia ME, Karagianni CHS (2017) Trituration and Fractal Dimension in Homeopathic Pharmacopoeia. Pharm Anal Acta

The image of $6 \mathrm{X}$ iron is red colored because the iron is black in SEM and the processing by NARO's software was impossible (Table 13).

\begin{tabular}{|l|l|l|l|l|l|l|l|l|}
\hline \multicolumn{1}{|l}{ Fractal dimention } \\
\hline Name & Arnica & Hypericum & Sod. chlor. & Benz. acid & Sulfur & Cal. phos. & Cal. carb. & Iron \\
\hline RSM & 19,606 & 19,606 & 19,606 & 19,606 & 19,606 & 19,606 & 19,754 & 19,606 \\
\hline $3 X$ & 17,021 & 16,957 & 17,057 & 16,964 & 14,315 & 14,472 & 16,475 & 12,618 \\
\hline $6 X$ & 14,785 & 14,124 & 14,757 & 14,694 & 13,825 & 12,757 & 14,613 & 12,464 \\
\hline
\end{tabular}

Table 13: The fractal dimension of all the different samples in different triturations.

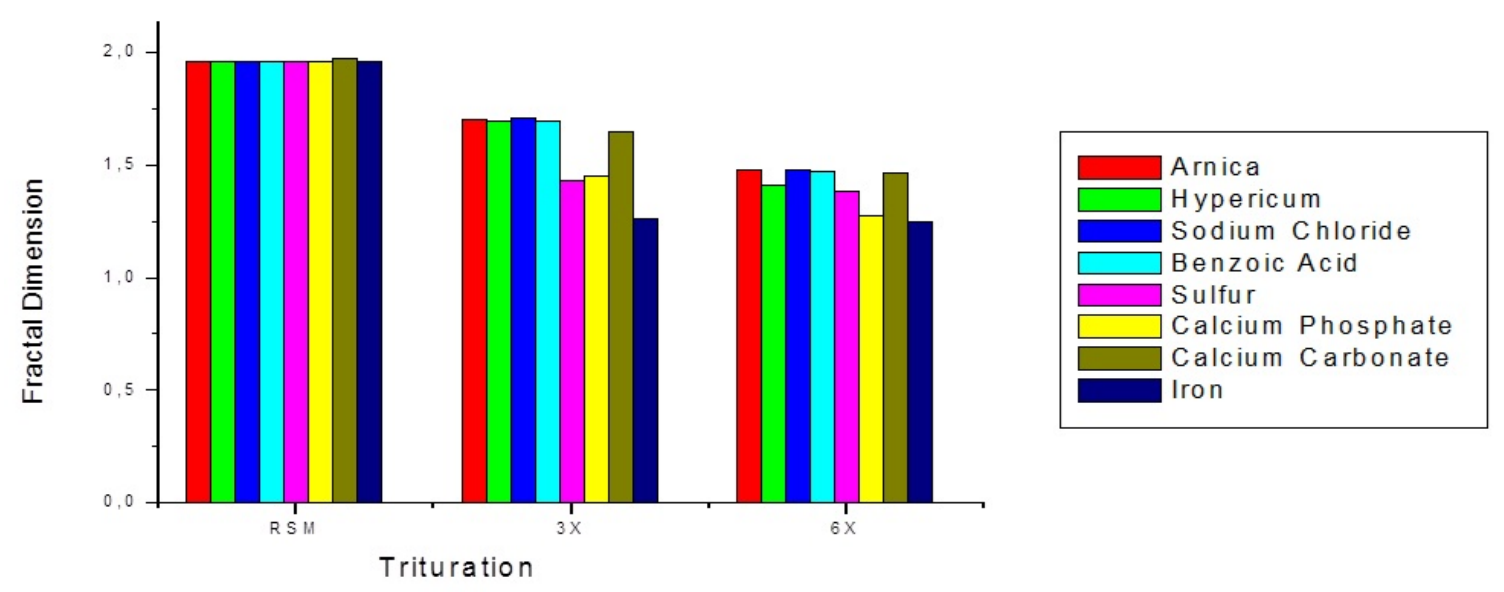

Figure 2: The fractal dimension of every sample in different triturations.

As we are checking the results of measurements in Figure 2 we see the variation of fractal dimensions of RSM as trituration ascend. Also, the values of the fractal dimensions decrease smoothly with increasing trituration. As it is observed in Figure 3, the dry plant samples, the salt, the organic acid and the oyster show a deviation of FD from $13-17 \%$ for $3 \mathrm{X}$ and $24-27 \%$ from $6 \mathrm{X}$ trituration. In minerals, we found that the deviation is $26-27 \%$ for $3 \mathrm{X}$ and $29-35 \%$ for $6 \mathrm{X}$. The iron's deviation appears to be $35.64 \%$ for $3 \mathrm{X}$ and $36.42 \%$ for $6 \mathrm{X}$ trituration. Also, it can be concluded that it is impossible to find universal correlations between the fractal dimensions of fractured surfaces and the conventional fracture parameters which are determined on the basis of the "straight-line crack" assumption. Moreover, one cannot use a fractal model to explain the fragmentation of the materials with respect to their toughness during trituration (Table 14).
Alternative and complementary therapy systems such as homeopathy have long been used around the world. Since 1995, homeopathy has been officially recognized in Europe as a system of medicine or a medical specialty. This is a therapeutic system that among the others, it uses solid natural substances as remedies after appropriate processing to make them soluble in water or ethyl alcohol. A process for achieving this in solids materials is the comminution.

Trituration is the term of several different methods used to process materials. In one sense, it is a form of comminution (reducing the particle size of a substance). In another sense, it is the production of a homogeneous material by mixing component materials thoroughly. Grinding in homeopathic pharmacopeia together with sugar of milk is a compulsory and necessary process that primarily aims at reducing the size of insoluble RSM to become soluble at the end of this process, so that solutions of homeopathic medicines can be prepared. 


\begin{tabular}{|l|l|l|l|l|l|l|}
\hline Name & FD-RSM & Change $\%$ & FD-3X & Change $\%$ & FD-6X & Change $\%$ \\
\hline Arnica & 19,606 & 0 & 17,021 & 13,18 & 14,785 & 24,58 \\
\hline Hypericum & 19,606 & 0 & 16,957 & 13,51 & 14,124 & 27,96 \\
\hline Sod. chlor. & 19,606 & 0 & 17,057 & 13,00 & 14,757 & 24,73 \\
\hline Benz. acid & 19,606 & 0 & 16,964 & 13,47 & 14,694 & 25,05 \\
\hline Sulfur & 19,606 & 0 & 14,315 & 26,98 & 13,825 & 29,48 \\
\hline Cal. phos. & 19,606 & 0 & 14,472 & 26,18 & 12,757 & 34,93 \\
\hline Cal. carb. & 19,754 & 0 & 16,475 & 16,59 & 14,613 & 26,02 \\
\hline Iron & 19,606 & 0 & 12,618 & 35,64 & 12,464 & 36,42 \\
\hline
\end{tabular}

Table 14: Fractal dimension change \% for every sample.

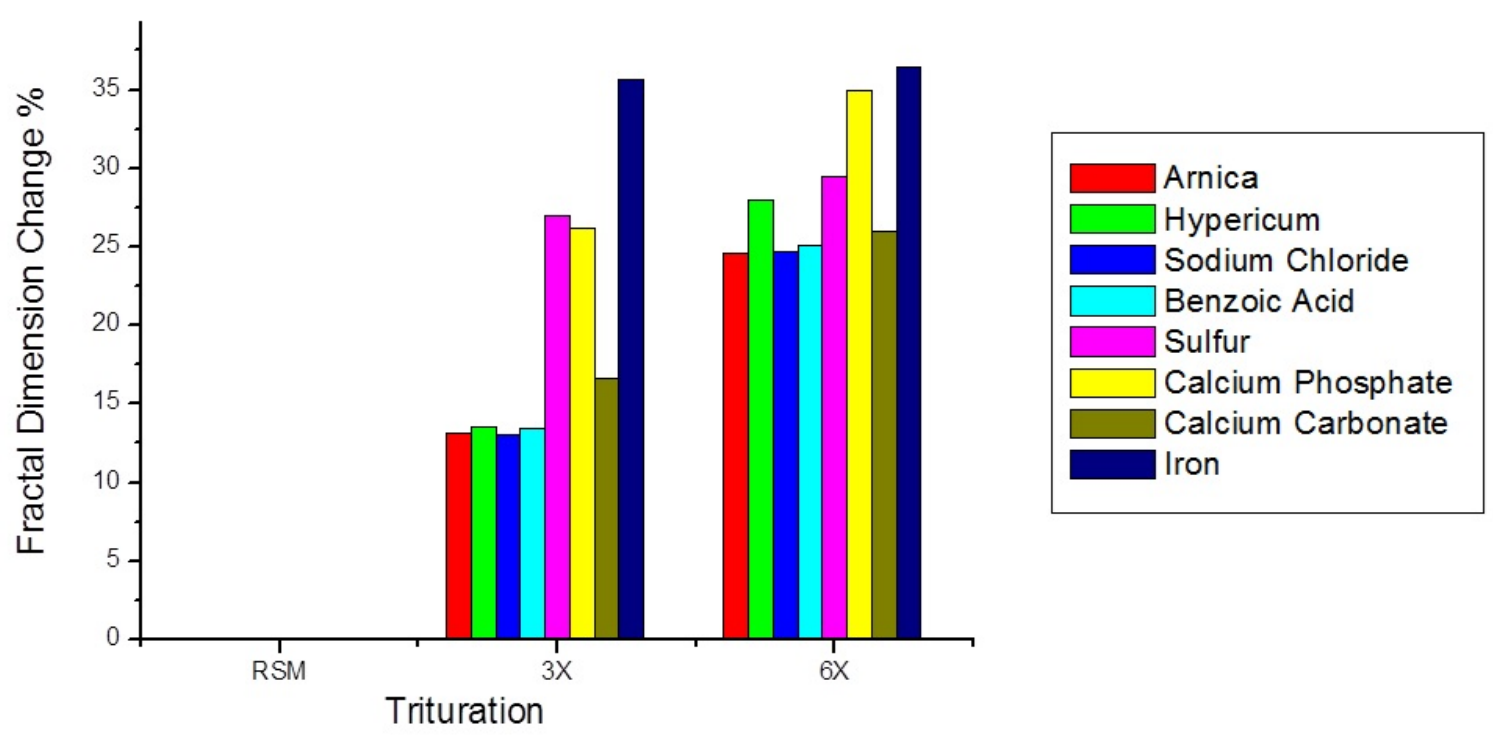

Figure 3: The fractal dimension change $\%$ for every sample in different triturations.

In this paper, the obtained results of the trituration as described in the homeopathic pharmacopoeia and has been proposed by Hahnemann and Hering are presented. It is in the framework of an exploratory work that examines the effect of the homeopathic solid RSM manufacturing process on the final products, before they are turned into solutions, considering these as common materials. The aim was to investigate which factors affect Hahnemann's trituration in the preparation of homeopathic drugs, since the grinding times are stable and independent of the type of materials. We have found that they seem to depend on the initial size of the materials. We showed that although our materials were different (metals, salts, plants, organic substances, etc.), with varying degrees of hardness, roughness, morphology etc., the grinding time itself no matter the origin or the original shape-size of our material (dust, chips, grains, etc.) seems to play a role in the final formulation of the product. This results in the preparation of homeopathic, original, much more homogeneous solutions, which will become remedies. This will help in the production of more quality homeopathic medicines, add new knowledge to their pharmaceutical preparations and homeopathic medicine will be established as a daily tool sure to be effective for the benefit of patients. 
What is more, the interest and the impact of this paper is to clarify the effect of trituration on the size-shape and, then, on materials' properties. The focus is especially on the homeopathic materials and on any other remedies, because trituration is used for preparation a lot of pharmaceutical products. We think that it is of great interest, for all the concerned disciplines, to know exactly what is achieved by trituration and why it is necessary to triturate the initial RSM. Our goal is to show what this process offers and how it may differentiate the final properties of the product, which may be of any remedy, homeopathic or not. The state of the art is to investigate the impact of each step and each intervention of the production process.

\section{Conclusion}

To sum up, these results conclude to the fact that the initial size and not the grain hardness of the starting RSM play a key role in the final shaping of the materials before turning them into solutions. These results indicate that the scaling properties of the fracture surface in trituration are not universal, but depend, albeit strongly, on materials grain sizes. Experimental and simulation results have indicated that the fractal character of fractured surfaces of materials, either natural or artificial, is ubiquitous [31]. It is indicated that the fractal dimension D is a measure of the entropy and, thus, it is also a state function [32]. This is the physical meaning of this approach. Theoretically, because of the established methodology of trituration, the randomness leads to structures having fractal dimensions that are always related to the initial state of RSM (hard, brittle, etc.) Respecting the scale, in the process of trituration a progressive division and diminution of the substance is affined. This mechanical subdivision gradually transforms in size the visible particles of the substance as the number of triturations ascend. It is obvious that all quantities used for both decimal and centesimal scale have measurable fractal dimensions. Moreover, since crack propagation of RSM during trituration is a nonreversible process, the entropy $S$ increases during the process, indicating from eq. (8) that the fractal dimension $\mathrm{D}$ of the fractured surfaces changes in the meantime, i.e. during trituration propagation process. Finally, fractal dimension D is believed to be a measure of entropy. By isolating a portion (initiator) from an isotropic mass (generator), with the same and always stereotypic formalism (recursive rule)-as it is applied in the preparation (trituration) of the classical homeopathic remedy, we managed to isolate one quantity which has fractal dimension, since in its entirety, is relatively self-affined, although the solid starting materials are different each time.

\section{Acknowledgments}

We would like to thank National Agriculture and Food Research Organization 3-1-1 Kannondai, Tsukuba, Ibaraki 305-8517, Japan for the gentle concession of software for fractal analysis for Windows and especially Dr. Hiroyuki Sasaki for support. Also, the pharmacists M. Filiano, A. Hatzopoulou, V. Kaliva for the gentle courtesy of various materials which are used in our research.

\section{References}

1. http://www.hpus.com/Draft-Guidelines-for-ManufacturingHomeopathic-Medicines.pdf

2. Boericke WA (1992) Compend of the principles of homoeopathy. B Jain Publishers Pvt Ltd., San Francisco Edition.

3. Pande CS, Richards LE, Louat N, Dempsey BD, Schwoebel AJ (1987) Fractal characterization of fractured surfaces Acra metal.
4. Arachovitis LI (2001) Introduction to chaotic dynamics and fractals. Papasotiriou Publications, Athens.

5. Mandelbrot B, Hudson RO (2006) The chart of chaos, translated by Elena pissia P. Travlos Publications, Athens.

6. Mandelbrot B (1982) The fractal geometry of nature. W.H. Freemand Co., NY, USA.

7. Feder J, Fractals (1988) Plenum. NY, USA.

8. Falkoner K (1990) Fractal geometry.

9. Frame M, Mandelbrot B (2001) "Fractals, Graphics, and Mathematics Education," Mathematical Association of America, Washington, DC, USA.

10. Mandelbrot BB, Passoja DE, Paullay AJ (1984) Fractal character of fracture surfaces of metals. Nature 308: 721-722.

11. Meisel LV (1991) Perimeter-area analysis, the slit-island method and the fractal characterization of metallic fracture surfaces. J Phy Appl Phys 24: 942-952.

12. http://www.gruppofrattura.it/ocs/index.php/esis/ECF13/paper/view/ $8593 / 5030$

13. Underwood EE, Banerji K (1986) Fractals in fractography. Mater Sci Eng 80: 1-14.

14. Kolednik O, Stüwe HP (1985) Engineering fracture mechanics.

15. Bryant D (1986) Micron Microscopy Acta 17: 237.

16. Kobayashi T, Shockey DA (1987) Metallurgical Transactions 18A, 1941.

17. Stampfl J, Scherer S, Gruber M, Kolednik O (1996) Applied Physics A63, 341.

18. Stampfl J, Scherer S, Berchthaler M, Gruber M, Kolednik O (1996) International Journal of Fracture 78: 35.

19. Milman VY, Stelmashenko NA, Blumenfe MR (1994) Fracture surfaces, a critical review of fractal studies and a novel morphological analysis of scanning tunneling microscopy measurements. Progress in Materials Science 38: 425-474.

20. Zhang X, Knackstedt MA, Chan DYC, Paterson L (1996) On the universality of fracture surface roughness Europhys. Lett 34: 121-126.

21. Boudis A (1995) Dynamic systems and chaos. Papasotiriou Publications, Athens p: 141-143.

22. Liu ST (2008).An improved differential box-counting approach to compute fractal dimension of gray-level image. Information Science and Engineering 1: 303-306.

23. Li J, Sun C, Du Q (2006) A new box-counting method for estimation of image fractal dimension. IEEE International Conference on Image Processing, pp: 3029-3032.

24. Lung CW (1986) Fractals and the fracture of cracked metals. In: Fractals in Physics Elsevier Science, Barking pp: 189-192.

25. Mu ZQ, Lung CW (1988) Studies on the fractal dimension and fracture toughness of steel. J. Phys. D: appl. Phys 21: 848-850.

26. Lung CW, Mu ZQ (1988) Fractal dimension measured with perimeterarea relation and toughness of materials. Phys Rev B 38: 781-784.

27. Negrepontis S, Zachariadis TH, Kalamidas N, Farmaki B (1997) General topology and equation analysis. Symmetria Publications, Athens p: 168.

28. Athanassopoulos D (1991) Theory of Probabilities. A Stamoulis Publications, Piraeus p: 44.

29. Vithoulkas G (2008) A new model for health and disease. Appendix $\mathrm{IACH}$.

30. Lu C (2013) Revisit on the fractal dimension in damage and fracture, 13th International Conference on Fracture, Beijing, China.

31. Lei W, Chen B (1995) Fractal characterization of some fracture phenomena. Engineering Fracture Mechanics 50: 149-155.

32. Kalliantas D (2008) The Chaos theory of disease. Kallianta A Publications, Eleusis p: 268. 